\title{
Two New Possible Mechanisms of Supernova-Like Explosions
}

\author{
V.V. Tikhomirov and S.E. Yuralevich \\ Institute for Nuclear Problems, Belarus State University, Bobrujskaya str. 11, \\ Minsk 220050, Belarus; \\ tikh@inp.minsk.by, svetaju@inp.minsk.by
}

Summary. Primordial black holes (PBHs) of microscopical size can completely absorb neutron stars (NSs) and white dwarfs (WDs) for less than the Hubble time. NS absorption is accompanied by inverse URCA process giving rise to emission of antineutrino. However considerable part of these antineutrino fails to escape NS being drawn into the growing black hole by accreting NS matter. The final stage of dense WD absorption is accompanied by $10^{51} \mathrm{erg}$ neutrino burst able to ignite nuclear burning giving rise to supernova-like WD explosion.

\section{Introduction}

Primordial black holes (PHBs) $[5,11]$ could form from primordial inhomogeneities, topological defects and phase transitions in the early Universe. Either PBH detection or proof of their nonexistence could give invaluable information about the earliest stages of cosmological expansion. Till present various methods of $\mathrm{PBH}$ search have been based on their Hawking radiation. These methods, however, have not led to detection of neither PBHs nor any consequence of their existence in the past, though stringent restrictions on their abundance have been established. That is why we would like to draw attention to an alternative way of $\mathrm{PBHs}$ search, namely to that based on PBHs interaction with cosmic objects, first of all with such dense ones as white dwarfs (WDs) and neutron stars (NSs). We will discuss the mechanisms of neutrino (antineutrino) emission by WD (NS) matter accreting onto a PBH, which can give rise to supernova like explosions. The neutrino burst accompanying WD absorption and igniting its nuclear burning is discussed in more detail.

\section{WD Absorption and Accompanying Neutrino Burst}

It is really possible to describe the process of WD matter accretion onto a PBH due to both the simplicity of degenerate electron gas equation of state and conservation of its adiabaticity under compression [7]. Right up to a growing PBH mass of about a hundredth of the WD's mass the equations [8] of spherical adiabatic collapse reduce to that of stationary spherical adiabatic accretion [10]. The latter allow to evaluate the WD matter density and 
velocity distributions, and to get the rate of WD matter accretion onto $\mathrm{BH}$ growing from a PBH and the time

$$
T_{W D}=\frac{27 \times 10^{9} y r}{\left(M_{b h} / 10^{15} g\right)\left(x_{0}+\sqrt{1+x_{0}^{2}}\right)^{3 / 2}}, \quad x_{0}=\left(\frac{\rho_{0}}{\rho_{r e l}}\right)^{1 / 3}
$$

of complete WD absorption by a PBH with initial mass $M_{b h}$. Here $\rho_{0}$ is central WD density in PBH absence and $\rho_{\text {rel }}=1.95 \times 10^{6} \mathrm{~g} / \mathrm{cm}^{3}$ is the density of carbon-oxygen WD separating nonrelativistic and ultrarelativistic ones. One can see that a WD of any density is absorbed by a PBH of mass $M \gg M_{*}$ for less than the Hubble time and that the most dense WDs with $1 \ll x_{0} \leq 10$ have enough time to be absorbed also by PBHs with $M \simeq M_{*}$. $M_{*}=5 \times 10^{14} \mathrm{~g}$ is the Hawking mass, PBHs of such mass have to complete their evaporation at present epoch.

WD absorption by $\mathrm{PBH}$ proves to be a really observable phenomenon due to accompanying neutrino emission. Indeed, the theory [10] allows to evaluate the maximum WD density reached at the growing $\mathrm{BH}$ surface $\rho_{\max } \simeq 1.44 \times 10^{11}\left(x_{0}+\sqrt{1+x_{0}^{2}}\right)^{1 / 2} \mathrm{~g} / \mathrm{cm}^{3}$. The electron Fermi energy amounts to $20-100 \mathrm{MeV}$ at such a density, thus, not only exceeding the threshold energies $\Delta=13.88 \mathrm{MeV}$ and $12.17 \mathrm{MeV}$ of the neutronization reactions

$$
{ }_{6}^{12} C+e^{-} \rightarrow_{5}^{12} B+\nu, \quad{ }_{5}^{12} B+e^{-} \rightarrow_{4}^{12} B e+\nu
$$

of carbon WD matter but also being sufficient to supply up to $\varepsilon_{\mathbf{F}}-\Delta \sim$ $10-100 \mathrm{MeV}$ to neutrino produced in these reactions. This way neutrino emission can provide a significant energy release on which a new approach of PBH search can be based.

Neutrino emission intensity grows with the accretion rate up to the moment of absorption of several tenth of WD mass, when the stationary accretion approximation is essentially violated and free fall of WD matter onto BH occurs. To describe the neutrino emission maximum one should use the adiabatic collapse theory [8] supplemented with the evolution equations

$$
\begin{array}{r}
\dot{n}=-n u^{\prime}-\frac{2 n u}{r}-n_{\mathrm{C}} \Gamma_{\mathrm{C}}-n_{\mathrm{B}} \Gamma_{\mathrm{B}}, \\
\dot{n}_{\mathrm{C}}=-n_{\mathrm{C}} u^{\prime}-\frac{2 n_{\mathrm{C}} u}{r}-n_{\mathrm{C}} \Gamma_{\mathrm{C}}, \\
\dot{n}_{\mathrm{B}}=-n_{\mathrm{B}} u^{\prime}-\frac{2 n_{\mathrm{B}} u}{r}+n_{\mathrm{C}} \Gamma_{\mathrm{C}}-n_{\mathrm{B}} \Gamma_{\mathrm{B}}
\end{array}
$$

of electron $(n)$, carbon $\left(n_{\mathrm{C}}\right)$ and boron $\left(n_{\mathrm{B}}\right)$ nuclei number densities at distance $r$ from the $\mathrm{BH}$ center. The nuclei neutronization probabilities $\Gamma_{\mathrm{C}, \mathrm{B}}=$ $\Gamma_{\mathrm{C}, \mathrm{B}}(n)$ are evaluated in the same way as that of proton [10]. Let us consider a case of absorption of a WD with central density $\rho_{0}=10^{9} \mathrm{~g} / \mathrm{cm}^{3}$ and mass $M_{W D}=2.68 \times 10^{33} \mathrm{~g}$ as an example. Its accretion density distributions at the moments of absorption of $0.01,0.04,0.16,0.32,0.64$ and 0.96 of $M_{W D}$ 


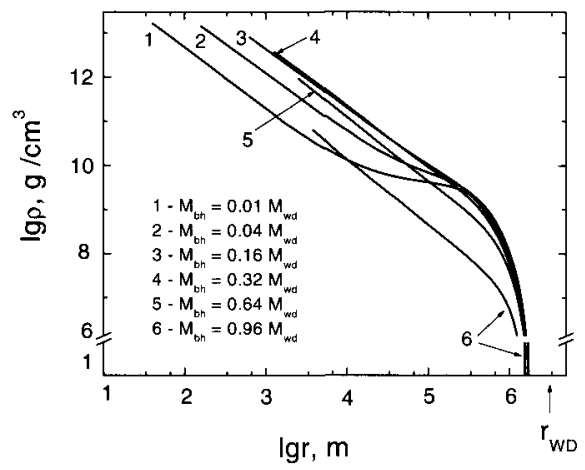

Fig. 1. Radial distributions of accreting WD matter density at the moments when $\mathrm{BH}$ mass reaches indicated parts of WD mass.

are given in Fig. 1. The neutrino emission intensity $I_{\nu}$ was evaluated taking into consideration the neutronization neutrino spectrum modified by both gravitational and kinematical Doppler effects as well as by neutrino capture by a growing $\mathrm{BH}$. Given in Fig. 2, time dependence of $I_{\nu}$ demonstrates that it reaches $(1-2) \times 10^{52} \mathrm{erg} / \mathrm{s}$ for about 0.1 second, taking away more then $10^{51} \mathrm{erg}$. The average neutrino energy exceeds $16 \mathrm{MeV}$ in this case. Note that the Doppler effects and neutrino capture lower together the total and average neutrino energy 3.5 and 1.65 times, respectively.

Such a neutrino burst can be detected by modern neutrino telescopes. In particular, SuperKamiokande will register about $300(k p c / r)^{2}$ neutrino events from such a burst at distance $r$, allowing to detect it if $r \leq 10 k p c$. A HyperKamiokande-scale telescope will be able to detect it in all the Galaxy.

It is quite possible that the WD absorption by a PBH can be observed, like a supernova explosions, at much greater distances. Though unable to simulate it in detail in the meanwhile, we can prove for sure that the described neutrino burst can ignite the nuclear burning. Indeed, the WD matter degeneracy, first, $Z+1$ times ( $Z$ is the nuclear charge) lowers its specific heat capacity and, second, nearly one order increases the $\nu-e$ cross-section [4]. Due to both of these factors the neutrino burst will be able to heat the massive WD layers up to $10^{10} \mathrm{~K}$ and more, quite enough [4] to ignite nuclear burning leading to a supernova-like explosion observable from other galaxies.

The absorption of more dense WDs with $\rho_{0}>10^{9} \mathrm{~g} / \mathrm{cm}^{3}$ will naturally be accompanied by even more intense neutrino emission and nuclear burning. However less dense absorbed WDs will emit much weaker. A WD with $\rho_{0}=$ $10^{8} \mathrm{~g} / \mathrm{cm}^{3}$ will emit only $1.4 \times 10^{49} \mathrm{erg}$ in the form of neutrino with average energy of only $4.6 \mathrm{MeV}$. It will be difficult to observe such a burst and it will hardly ignite nuclear burning. To find a threshold WD density of thermonuclear ignition and simulate the nuclear burning will be our next step. 


\section{NS Absorption}

One can foresee that as more dense objects NSs will be absorbed by PBHs much faster than WDs. In fact, Hawking predicted that as in early as his paper [5]. Our estimates show that a PBH of Hawking mass will be absorbed for a few million years and that the absorption time is inversely proportional to PBH initial mass. Thus there is no doubt that a NS will have enough time to be absorbed by a PBH and the possibility to observe this phenomenon is entirely determined by the existence and efficiency of a mechanism of energy release by accreting NS matter.

Using various equations of state of NS matter $[9,10]$ we have elucidated that the degree of compression of accreting NS matter will hardly exceed ten and, thus, will not give rise to the results of [3]. The actual mechanism of energy release by compressed NS matter will be modified URCA process driven at zero temperature by the neutron energy increase which accompanies compression. A spectrum of antineutrino specific emission capacity can be evaluated following [10]

$$
\begin{aligned}
\frac{d N_{\tilde{\nu}}}{d \varepsilon_{\tilde{\nu}} d t}= & \frac{m_{n}^{3} m_{p}^{3 / 2} \varepsilon_{\tilde{\nu}}^{2}}{2^{12} c^{6} \hbar^{4}} \int_{\varepsilon_{F p}}^{\varepsilon_{F n}-\varepsilon_{F e}+q-\varepsilon_{\tilde{\nu}}} \int_{\varepsilon_{F e}}^{\varepsilon_{F n}+q-\varepsilon_{p}-\varepsilon_{\tilde{\nu}}} \int_{\varepsilon_{e}+\varepsilon_{p}+\varepsilon_{\bar{\nu}-q}}^{\varepsilon_{F n}} \\
& \int_{\varepsilon_{F n}-q-\varepsilon_{n 1}+\varepsilon_{p}+\varepsilon_{e}+\varepsilon_{\tilde{\nu}}}^{\varepsilon_{F n}} \sum_{\text {spins }}\left|H_{f i}\right|^{2} \sqrt{\varepsilon_{p}} \varepsilon_{e}^{2} d \varepsilon_{p} d \varepsilon_{e} d \varepsilon_{n 1} d \varepsilon_{n 2}
\end{aligned}
$$

where $\sum_{\text {spins }}\left|H_{f i}\right|^{2}$ is the squared matrix element of the reaction

$$
n+n \rightarrow n+p+e^{-}+\tilde{\nu}
$$

sum over final and averaged over initial particle spins, $q=\left(m_{n}-m_{p}\right) c^{2}, m_{n}$ and $m_{p}$ are the neutron and proton masses, $\varepsilon_{\tilde{\nu}}, \varepsilon_{n 1}, \varepsilon_{n 2}, \varepsilon_{p}$ and $\varepsilon_{e}$ are antineutrino, two neutron, proton and electron energies respectively. According to Eq. 4 the average antineutrino energy amounts to few tens MeV. Similarly to the WD case, the most part of antineutrino energy will be released at the stage of free fall of NS matter onto a $\mathrm{BH}$ having the mass comparable with that of NS. Since the free fall time does not exceed the time of antineutrino diffusion to the neutrinosphere, a considerable, if not the most part of emitted antineutrino will be drawn into the $\mathrm{BH}$ with accreting matter. This circumstance allows to argue that NS absorption will not be accompanied by such large energy release as that of a WD. It should be also mensioned that both WD and NS absorption by PBHs will give rise to formation of $\mathrm{BH}$ which are essentially lighter than that forming in usual star collapse.

\section{PBH Caption}

The best condition for an efficient gravitational PBH capture existed at the epoch preceding the galaxy formation when the first astrophysical objects 


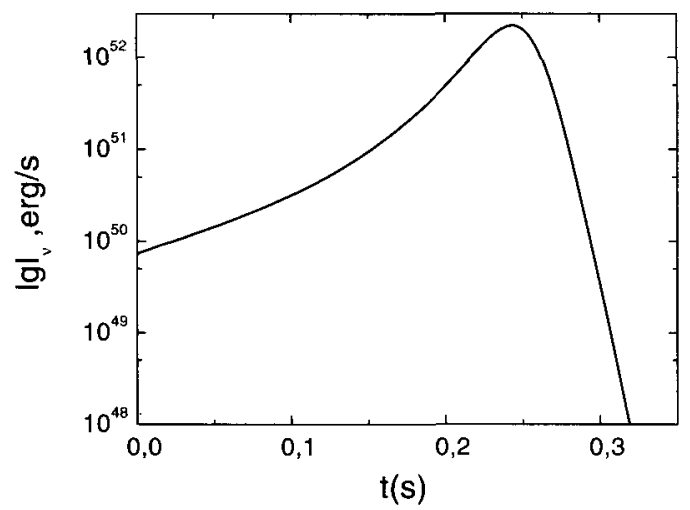

Fig. 2. Time dependence of a neutrino emission intensity measured from the moment when $M_{b h}=0.01 M_{W D}$.

with masses of the order $10^{5}-10^{6} M_{\odot}$ were forming [2]. A PBH capture by first solitary stars [1] looks the most promising. However except for the formation process, further evolution of such stars has not been investigated and does not allow to estimate the number of WDs and NSs formed. Globular clusters (GCs) will also abundantly capture PBHs. Both the high concentration and low velocity of stars as well as the high concentration of captured PBHs in GCs predetermine a high probability of PBH capture by stars in triple PBHstar-star collisions. Further process of star binary hardening in collisions and companion exchange with other stars lead to significant $\mathrm{PBH}$ orbit shrinking and penetration of considerable PBH fraction into stars, some of which turn later into WDs and NSs. Our investigation of these processes is in progress.

\section{Conclusions}

In conclusion, complete WD absorption by a $\mathrm{PBH}$ can give rise to a supernova like neutrino burst which is able to ignite WD matter nuclear burning and opens up a new way of PBH detection.

\section{References}

1. T. Abel, G.L. Bryan, M.L. Norman: Science 295, 93 (2002)

2. E.V. Derishev, A.A. Belyanin: Astrophys. Space Sci. 343, 1 (1999)

3. E.V. Derishev, V.V. Kocharovsky, Vl.V. Kocharovsky: Pisma v Zh. Eksp. Teor. Fiz. 70, 652 (1999)

4. S.S. Gershtein et al. : Zh. Eksp. Teor. Fiz. 69, 1473 (1975)

5. S.W. Hawking: Mon. Not. R. Astron. Soc. 152, 75 (1972)

6. D.C. Heggie: Mon. Not. R. Astron. Soc. 173, 729 (1975) 
7. S.E. Juralevich, V.V. Tikhomirov: astro-ph 0202445

8. C.W. Misner, D.H. Sharp: Astrophys. Space Sci. 136, 571 (1964)

9. G.S. Sahakian: In: Physics of Neutron Stars, (JINR, Dubna 1995) pp. 45-56

10. S. Shapiro, S. Teukolsky: In: Black Holes, White Dwarfs and Neutron Stars, (Wiley, New York 1986) p. 176

11. J.B. Zel'dovich, I.D. Novikov: Astron. Zhurnal 43, 758 (1966) 